\title{
No stable static spherically symmetric wormholes in Horn- deski theory
}

\author{
Oleg Evseev ${ }^{1,2, *}$ and Oleg Melichev ${ }^{1,2, * *}$ \\ ${ }^{1}$ Institute for Nuclear Research of the Russian Academy of Sciences, 60th October Anniversary \\ Prospect 7a, Moscow, 117312, Russia \\ ${ }^{2}$ Department of Particle Physics and Cosmology, Physics Faculty, M. V. Lomonosov Moscow State \\ University, Vorobyovy Gory, 1-2, Moscow 119991, Russia
}

\begin{abstract}
We consider the most general theory of a single scalar field with the second order field equations, the Horndeski theory, in four-dimensional space-time. We show that static, spherically symmetric, asymptotically flat, Lorentzian wormholes are unstable in this theory.
\end{abstract}

\section{Introduction}

Wormholes [1-6] and semiclosed worlds [7-10] are spatial geometries with a throat which connects either two flat spaces or a flat space and a closed world, respectively.
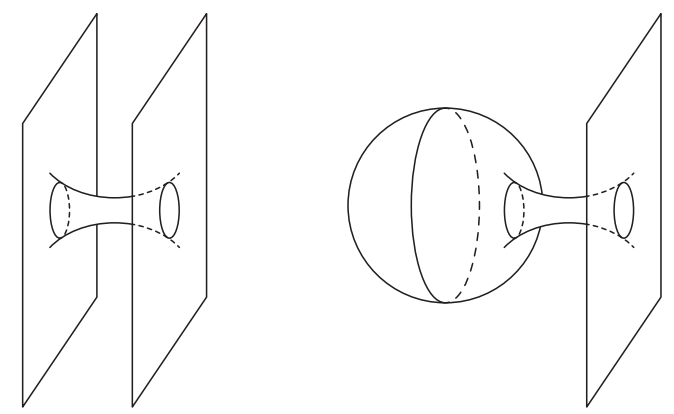

Figure 1. A wormhole (on the left) and a semiclosed world (on the right). The static case; the third spatial dimension is omitted.

If they are traversable, they both have the trapped surface (see figure 1). This, combined with the noncompactness of the Cauchy hypersurface and the Null Energy Condition (NEC):

$$
G_{\mu \nu} \eta^{\mu} \eta^{\nu} \geq 0 \text { for any null vector } \eta^{\mu},
$$

where $G_{\mu \nu}=R_{\mu \nu}-\frac{1}{2} R g_{\mu \nu}$ is the Einstein tensor, leads to the singularity in finite future: this is the result of the Penrose theorem [11]. The condition (1) for matter minimally coupled to

*e-mail: oa.evseev@physics.msu.ru

**e-mail: oi.melichev@physics.msu.ru 
gravity reads

$$
T_{\mu \nu} \eta^{\mu} \eta^{v} \geq 0 \text { for any null vector } \eta^{\mu},
$$

where $T_{\mu \nu}$ is the energy-momentum tensor of matter. The NEC is hard to violate; it holds for all known forms of matter.

According to the Penrose theorem [11], there is a theoretical opportunity to construct a stable static spherically symmetric wormhole in theories violating NEC. We consider the most general scalar field theory with second-order field equations, the Horndeski theory (generalised Galileons plus gravity):

$$
S=\int d^{4} x\left(\mathcal{L}_{2}+\mathcal{L}_{3}+\mathcal{L}_{4}+\mathcal{L}_{5}\right)
$$

$$
\begin{aligned}
& \mathcal{L}_{2}=K(\phi, X), \\
& \mathcal{L}_{3}=-G_{3}(\phi, X) \square \phi, \\
& \mathcal{L}_{4}=G_{4}(\phi, X) R+G_{4 X}\left[(\square \phi)^{2}-\left(\nabla_{\mu} \nabla_{\nu} \phi\right)^{2}\right], \\
& \mathcal{L}_{5}=G_{5}(\phi, X) G_{\mu \nu} \nabla^{\mu} \nabla^{\nu} \phi-\frac{1}{6} G_{5 X}\left[(\square \phi)^{3}-3 \square \phi\left(\nabla_{\mu} \nabla_{\nu} \phi\right)^{2}+2\left(\nabla_{\mu} \nabla_{\nu} \phi\right)^{3}\right],
\end{aligned}
$$

where $X=-\frac{1}{2} \nabla_{\mu} \phi \nabla^{\mu} \phi, \square \phi=\nabla_{\mu} \nabla^{\mu} \phi, R$ is the scalar curvature, $G_{\mu v}$ is the Einstein tensor and metric signature is $(-,+,+,+)$.

This class of theories is known to have second-order field equations despite the secondorder derivatives in the Lagrangian. The most general scalar field theory with such property was first derived by Horndeski in 1974 [12] but stayed unnoticed until 1992 when it was reintroduced by Fairlie, Govaerts and Morozov [13-15] and became popular quite recently [16-32]. The point is that Horndeski theories are capable, at least in principle, of violating the NEC without obvious pathologies.

It has been shown, however, that both asymptotically flat, static, spherically symmetric wormholes [33, 34] and semiclosed worlds [35] are unstable in theories with $G_{4}=M_{P l}^{2} / 2=$ const, $G_{5}=0$.

The purpose of this paper is to extend this result to wormholes in the most general Horndeski theory (3). The proof of the analogous (by interchanging time and radial coordinate) no-go theorem for bouncing cosmologies within $G_{3}$ theories plus gravity was given in [36] and generalised to the case of the interaction of the Galileon field with an extra scalar field in [37]. Afterwards, this proof was further extended to the full Horndeski theory in [38] and the multi-Galileon Horndeski theory in [39].

The paper is organised as follows. We give a brief review of the essential for our argument results obtained by Kobayashi, Motohashi and Suyama [40, 41] in Sec. 2, considering both odd (Subsec. 2.1) and even (Subsec. 2.2) sectors. Finally, we prove the instability in Sec. 3.

\section{Stability conditions}

The configuration to consider is static and spherically symmetric, so we use the most general form of metric for that case:

$$
d s^{2}=-a^{2}(r) d t^{2}+\frac{d r^{2}}{b^{2}(r)}+c^{2}(r)\left(d \theta^{2}+\sin ^{2} \theta d \varphi^{2}\right)
$$


and use the gauge

$$
a(r)=b(r) .
$$

The asymptotic behaviour of metric functions as $r \rightarrow \pm \infty$ is the following:

$$
a(r) \rightarrow 1, \quad c(r) \rightarrow|r|
$$

As we are working with the spherically symmetric Ansatz, we can now consider the decomposition of any scalar under the rotations of a $2 \mathrm{D}$ sphere in spherical harmonics $Y_{l}^{m}(\theta, \varphi)$ :

$$
s(t, r, \theta, \varphi)=\sum_{l=0}^{\infty} \sum_{m=-l}^{l} s_{l m}(t, r) Y_{l}^{m}(\theta, \varphi)
$$

Any vector $V_{a}$ and symmetric tensor $T_{a b}$ can be decomposed as follows:

$$
\begin{aligned}
& V_{a}(t, r, \theta, \varphi)=\stackrel{\gamma}{\nabla} \Phi_{a} \Phi_{1}(t, r, \theta, \varphi)+E_{a}^{b} \nabla_{b} \Phi_{2}(t, r, \theta, \varphi), \\
& T_{a b}(t, r, \theta, \varphi)=\stackrel{\gamma}{\nabla_{a}} \nabla_{b} \Psi_{1}+\gamma_{a b} \Psi_{2}(t, r, \theta, \varphi) \\
& +\frac{1}{2}\left(E_{a}^{c} \nabla_{c}^{\gamma} \nabla_{b}^{\gamma} \Psi_{3}(t, r, \theta, \varphi)+E_{b}^{c} \stackrel{\gamma}{\nabla}_{c} \nabla_{a} \Psi_{3}(t, r, \theta, \varphi)\right) .
\end{aligned}
$$

$\gamma_{a b}$ here denotes the metric on $S^{2} ; \stackrel{\gamma}{\nabla}_{a}$ is the covariant derivative ${ }^{1}$ for $\gamma_{a b} ; E_{a b}=\sqrt{\operatorname{det} \gamma} \varepsilon_{a b}$, $\varepsilon_{a b}$ is the Levi-Civita symbol, $\varepsilon_{\theta \varphi}=1 ; \Phi_{1}, \Phi_{2}, \Psi_{1}, \Psi_{2}, \Psi_{3}$ are arbitrary scalar functions; $Y_{l}^{m}(\theta, \varphi)$ are spherical harmonics. We can now replace $\Phi_{1}, \Phi_{2}, \Psi_{1}, \Psi_{2}$ and $\Psi_{3}$ with the corresponding decompositions using (7) and obtain all scalars, vectors and symmetric secondorder tensors in terms of spherical harmonics.

Notice that terms with $E_{a b}$ get a factor $(-1)^{l}$ under parity transformation $(\theta, \varphi) \rightarrow(\pi-$ $\theta, \pi+\varphi)$ while terms without $E_{a b}$ get a factor $(-1)^{l+1}$ stipulating the names of modes: they are called even- and odd-parity modes, respectively.

\subsection{Odd-parity sector}

Odd-parity perturbations (terms with $E_{a b}$ ) read

$$
\begin{aligned}
\delta \phi & =0, \quad h_{t t}=0, \quad h_{t r}=0, \quad h_{r r}=0, \\
h_{t a} & =\sum_{l=0}^{\infty} \sum_{m=-l}^{l} h_{0, l m}(t, r) E_{a b} \partial^{b} Y_{l}^{m}(\theta, \varphi), \\
h_{r a} & =\sum_{l=1}^{\infty} \sum_{m=-l}^{l} h_{1, l m}(t, r) E_{a b} \partial^{b} Y_{l}^{m}(\theta, \varphi), \\
h_{a b} & =\frac{1}{2} \sum_{l=2}^{\infty} \sum_{m=-l}^{l} h_{2, l m}(t, r)\left[E_{a}^{c} \stackrel{\gamma}{\nabla}_{c} \stackrel{\gamma}{\nabla}_{b} Y_{l}^{m}(\theta, \varphi)+E_{b}^{c} \stackrel{\gamma}{\nabla}_{c} \stackrel{\gamma}{\nabla}_{a} Y_{l}^{m}(\theta, \varphi)\right] .
\end{aligned}
$$

The components of gauge functions in the odd-partity sector are

$$
\xi_{t}=0, \quad \xi_{r}=0, \quad \xi_{a}=\sum_{l=0}^{\infty} \sum_{m=-l}^{l} \Lambda_{l m}(t, r) E_{a b} \partial^{b} Y_{l}^{m}(\theta, \varphi)
$$

\footnotetext{
${ }^{1}$ The symbol ${ }_{\nabla}^{\gamma}$ should not be confused with $\nabla_{\mu}$, the covariant derivative for $g_{\mu \nu}$.
} 
We make use of this gauge freedom to eliminate unphysical degrees of freedom for $l \geq 2$ (Regge-Wheeler approach, $m$ is set equal to zero without loss of generality, see [40, 42] for details) and obtain the following Lagrangian for perturbations:

$$
\mathcal{L}^{(2)}=\frac{l(l+1)}{4(l-1)(l+2)}\left[\mathcal{A} \dot{q}^{2}-\mathcal{B} q^{\prime 2}-l(l+1) C q^{2}-V(r) q^{2}\right]
$$

where

$$
\mathcal{A}=\frac{c^{2}}{a^{2}} \frac{\mathcal{H}^{2}}{\mathcal{G}}, \quad \mathcal{B}=a^{2} c^{2} \frac{\mathcal{H}^{2}}{\mathcal{F}}, \quad C=a^{2} \mathcal{H}, \quad q=\dot{h}_{1}-h_{0}^{\prime}+2 \frac{c^{\prime}}{c} h_{0},
$$

and

$$
\begin{aligned}
& \mathcal{F}=2\left(G_{4}+\frac{a^{2}}{2} \phi^{\prime} X^{\prime} G_{5 X}-X G_{5 \phi}\right), \\
& \mathcal{G}=2\left[G_{4}-2 X G_{4 X}+X\left(a a^{\prime} c^{\prime} \phi^{\prime} G_{5 X}+G_{5 \phi}\right)\right], \\
& \mathcal{H}=2\left[G_{4}-2 X G_{4 X}+X\left(a^{2} \frac{c^{\prime}}{c} \phi^{\prime} G_{5 X}+G_{5 \phi}\right)\right] .
\end{aligned}
$$

Here the background field equations are used, $V(r)$ is the effective potential, which is irrelevant for our purposes, $C$-part corresponds to wave propagation along the angular direction. To obtain this Lagrangian, $h_{0}$ and $h_{1}$ were treated as auxiliary fields and were eliminated.

The stability conditions for this Ansatz (fist obtained by Kobayashi, Motohashi and Suyama [40]) are

$$
\begin{aligned}
\mathcal{F} & >0 \text { for the stability along the radial direction, } \\
\mathcal{G} & >0 \text { for abscense of ghosts, } \\
\mathcal{H} & >0 \text { for the stability along the angular directions. }
\end{aligned}
$$

\subsection{Even-parity sector}

The perturbations in the even-parity sector (terms without $E_{a b}$ ) read

$$
\begin{aligned}
& \delta \phi=\sum_{l=0}^{\infty} \sum_{m=-l}^{l} \delta \phi_{l m}(t, r) Y_{l}^{m}(\theta, \varphi), \quad h_{t t}=a^{2} \sum_{l=0}^{\infty} \sum_{m=-l}^{l} H_{0, l m}(t, r) Y_{l}^{m}(\theta, \varphi), \\
& h_{t r}=\sum_{l=0}^{\infty} \sum_{m=-l}^{l} H_{1, l m}(t, r) Y_{l}^{m}(\theta, \varphi), \quad h_{r r}=\frac{1}{a^{2}} \sum_{l=0}^{\infty} \sum_{m=-l}^{l} H_{2, l m}(t, r) Y_{l}^{m}(\theta, \varphi) \text {, } \\
& h_{t a}=\sum_{l=0}^{\infty} \sum_{m=-l}^{l} \beta_{l m} \partial_{a} Y_{l}^{m}(\theta, \varphi), \quad h_{r a}=\sum_{l=0}^{\infty} \sum_{m=-l}^{l} \alpha_{l m} \partial_{a} Y_{l}^{m}(\theta, \varphi), \\
& h_{a b}=c^{2} \sum_{l=0}^{\infty} \sum_{m=-l}^{l}\left[K_{l m}(t, r) \gamma_{a b} Y_{l}^{m}(\theta, \varphi)+G_{l m}(t, r) \stackrel{\gamma}{\nabla_{a}} \nabla_{b} Y_{l}^{m}(\theta, \varphi)\right] \text {. }
\end{aligned}
$$

Note that in the even-parity sector, the scalar field is perturbed as well. We use the same procedure to fix the gauge and eliminate unphysical degrees of freedom (see details in [41, $42]$ ) and derive the following Lagrangian for perturbations (once again, $l \geq 2$ ):

$$
\mathcal{L}=\frac{1}{2} \mathcal{K}_{i j} \dot{v}^{i} \dot{v}^{j}-\frac{1}{2} \mathcal{G}_{i j} v^{i^{\prime}} v^{j^{\prime}}-\frac{1}{2} Q_{i j} v^{i} v^{j^{\prime}}-\frac{1}{2} \mathcal{M}_{i j} v^{i} v^{j}
$$


where $i=\overline{1,2}, j=\overline{1,2} ; v^{1} \equiv \psi, v^{2} \equiv \delta \phi$. Expressions for $\mathcal{K}_{i j}, \mathcal{G}_{i j}, Q_{i j}$ and $\mathcal{M}_{i j}$ are cumbersome; we write their combinations explicitly whenever we need them.

The absence of ghost is guaranteed when $\mathcal{K}_{11}>0$ and $\operatorname{det} \mathcal{K}>0$; we need the latter condition:

$$
\operatorname{det} \mathcal{K}=\frac{4(l-1)(l+2)\left(2 c c^{\prime} \mathcal{H}+\Xi \phi^{\prime}\right)^{2} \mathcal{F}\left(2 \mathcal{P}_{1}-\mathcal{F}\right)}{l(l+1) a^{4} \mathcal{H}^{2} \phi^{\prime 2}\left(2 c c^{\prime} \mathcal{H}+\Xi \phi^{\prime}\right)^{2}}>0,
$$

where

$$
\mathcal{P}_{1}=\frac{\left(2 c c^{\prime} \mathcal{H}+\Xi \phi^{\prime}\right)}{2 c^{2} \mathcal{H}^{2}} \cdot \frac{d}{d r}\left[\frac{c^{4} \mathcal{H}^{4}}{\left(2 c c^{\prime} \mathcal{H}+\Xi \phi^{\prime}\right)^{2}}\right],
$$

and $\Xi$ is defined as follows:

$$
\begin{aligned}
\Xi= & 2 c^{2}\left[-X G_{3 X}+2 a^{2} \frac{c^{\prime}}{c} \phi^{\prime}\left\{G_{4 X}+2 X G_{4 X X}-\left(X G_{5 \phi}\right)_{X}\right\}\right. \\
& \left.+G_{4 \phi}+2 X G_{4 \phi X}-\frac{1}{c^{2}} X G_{5 X}+a^{2} \frac{c^{\prime 2}}{c^{2}}\left(3 X G_{5 X}+2 X^{2} G_{5 X X}\right)\right] .
\end{aligned}
$$

In view of (20), the condition (24) becomes

$$
2 \mathcal{P}_{1}-\mathcal{F}>0
$$

\section{No-go theorem}

The stability conditions important for our purposes are (17) and (19) from the odd-parity sector and (26) from the even-parity sector. We introduce the new variable

$$
Q=\frac{2 c c^{\prime} \mathcal{H}+\Xi \phi^{\prime}}{c^{2} \mathcal{H}^{2}}
$$

and integrate the inequality (26) from $r$ to $r^{\prime}>r$. In this way we obtain the stability condition in the following form:

$$
Q^{-1}(r)-Q^{-1}\left(r^{\prime}\right)<-\frac{1}{2} \int_{r}^{r^{\prime}} \mathcal{F} d r .
$$

Let $Q^{-1}(r)$ be negative at some $r$. Then we rewrite the inequality (28) as

$$
Q^{-1}\left(r^{\prime}\right)>Q^{-1}(r)+\frac{1}{2} \int_{r}^{r^{\prime}} \mathcal{F} d r
$$

and notice that if the integral in the right-hand side diverges as $r^{\prime} \rightarrow+\infty$, then $Q^{-1}\left(r^{\prime}\right)$ is positive at large $r^{\prime}$, meaning that $Q$ is singuar at some point $r^{*}$.

Let $Q^{-1}\left(r^{\prime}\right)$ be positive at some $r^{\prime}$. Then we rewrite the inequality (28) as

$$
Q^{-1}(r)<Q^{-1}\left(r^{\prime}\right)-\frac{1}{2} \int_{r}^{r^{\prime}} \mathcal{F} d r
$$

If the integral in the right-hand side diverges as $r \rightarrow-\infty$ then $Q^{-1}(r)$ is negative at large negative $r$, meaning that $Q$ is singuar at some point $r^{*}$. 
We now notice that the requirement that general relativity is restored away from the wormhole gives

$$
\left\{\begin{array}{l}
G_{4} \rightarrow M_{P l}^{2} / 2 \\
G_{5} \rightarrow 0
\end{array} \quad \text { as } r \rightarrow \pm \infty,\right.
$$

leading to $\mathcal{F} \rightarrow M_{P l}^{2}$ and thus to the divergent integrals in the right-hand sides of (29) and (30), as $r^{\prime} \rightarrow+\infty$ and $r \rightarrow-\infty$, respectively. This means that $Q$ has to be singular. We now take a closer look at the definition of $Q$, Eq. (27), and notice that:

- $c$ is bounded from below and $\mathcal{H}>0$, Eq. (22), so the denominator is non-zero for any $r$;

- $c^{\prime}$ is non-singular and if $\mathcal{H}$ diverges, then $Q \rightarrow 0$, not $Q \rightarrow \infty$;

- thus, singular $Q$ requires either singular $\Xi$ or singular $\phi^{\prime}$ or both; in either case the solution is singular.

These statements complete the argument.

\section{Acknowledgements}

The authors are indebted to V. Rubakov, S. Mironov and V. Volkova for helpful discussions. This work has been supported by Russian Science Foundation Grant No. 14-22-00161.

\section{References}

[1] M.S. Morris, K.S. Thorne, Am. J. Phys. 56, 395 (1988)

[2] M.S. Morris, K.S. Thorne, U. Yurtsever, Phys. Rev. Lett. 61, 1446 (1988)

[3] M. Visser, Lorentzian wormholes: From Einstein to Hawking (1995), ISBN 1563966530, 9781563966538

[4] D. Hochberg, M. Visser, Phys. Rev. D58, 044021 (1998), gr-qc/9802046

[5] I.D. Novikov, N.S. Kardashev, A.A. Shatskiy, Phys. Usp. 50, 965 (2007), [Usp. Fiz. Nauk177,1017(2007)]

[6] A. Shatskiy, I.D. Novikov, N.S. Kardashev, Phys. Usp. 51, 457 (2008), 0810.0468

[7] V.P. Frolov, M.A. Markov, V.F. Mukhanov, Phys. Rev. D41, 383 (1990)

[8] E.I. Guendelman, Int. J. Mod. Phys. D19, 1357 (2010), 1003. 3975

[9] S.V. Chernov, V.I. Dokuchaev, Class. Quant. Grav. 25, 015004 (2008), 0709. 0616

[10] V.I. Dokuchaev, S.V. Chernov, JETP Lett. 85, 595 (2007), [Pisma Zh. Eksp. Teor. Fiz.85,727(2007)], 1208.5249

[11] R. Penrose, Phys. Rev. Lett. 14, 57 (1965)

[12] G.W. Horndeski, Int. J. Theor. Phys. 10, 363 (1974)

[13] D.B. Fairlie, J. Govaerts, A. Morozov, Nucl. Phys. B373, 214 (1992), hep-th/9110022

[14] D.B. Fairlie, J. Govaerts, Phys. Lett. B281, 49 (1992), hep-th/9202056

[15] D.B. Fairlie, J. Govaerts, J. Math. Phys. 33, 3543 (1992), hep-th/9204074

[16] G.R. Dvali, G. Gabadadze, M. Porrati, Phys. Lett. B485, 208 (2000), hep-th/0005016

[17] M.A. Luty, M. Porrati, R. Rattazzi, JHEP 09, 029 (2003), hep-th/0303116

[18] A. Nicolis, R. Rattazzi, JHEP 06, 059 (2004), hep-th/0404159

[19] A. Nicolis, R. Rattazzi, E. Trincherini, Phys. Rev. D79, 064036 (2009), 0811 . 2197

[20] C. Deffayet, G. Esposito-Farese, A. Vikman, Phys. Rev. D79, 084003 (2009), Q901. 1314

[21] C. de Rham, A.J. Tolley, JCAP 1005, 015 (2010), 1003. 5917 
[22] G. Goon, K. Hinterbichler, M. Trodden, Phys. Rev. Lett. 106, 231102 (2011), 1103.6029

[23] G. Goon, K. Hinterbichler, M. Trodden, JCAP 1107, 017 (2011), 1103.5745

[24] K. Kamada, T. Kobayashi, M. Yamaguchi, J. Yokoyama, Phys. Rev. D83, 083515 (2011), 1012.4238

[25] T. Kobayashi, M. Yamaguchi, J. Yokoyama, Prog. Theor. Phys. 126, 511 (2011), 1105.5723

[26] E. Babichev, C. Deffayet, R. Ziour, Int. J. Mod. Phys. D18, 2147 (2009), 0905 . 2943

[27] C. Deffayet, O. Pujolas, I. Sawicki, A. Vikman, JCAP 1010, 026 (2010), 1008.0048

[28] T. Kobayashi, M. Yamaguchi, J. Yokoyama, Phys. Rev. Lett. 105, 231302 (2010), 1008.0603

[29] O. Pujolas, I. Sawicki, A. Vikman, JHEP 11, 156 (2011), 1103.5360

[30] C. Charmousis, E.J. Copeland, A. Padilla, P.M. Saffin, Phys. Rev. D85, 104040 (2012), 1112.4866

[31] R. Kolevatov, Phys. Rev. D92, 123532 (2015), 1508.00046

[32] R. Kolevatov, S. Mironov, V. Rubakov, N. Sukhov, V. Volkova, Phys. Rev. D96, 125012 (2017), 1708.04262

[33] V.A. Rubakov, Teor. Mat. Fiz. 187, 338 (2016), [Theor. Math. Phys.187,no.2,743(2016)], 1509.08808

[34] V.A. Rubakov, Theor. Math. Phys. 188, 1253 (2016), [Teor. Mat. Fiz.188,no.2,337(2016)], 1601.06566

[35] O.A. Evseev, O.I. Melichev, Phys. Rev. D96, 024030 (2017), 1607.01721

[36] M. Libanov, S. Mironov, V. Rubakov, JCAP 1608, 037 (2016), 1605. 05992

[37] R. Kolevatov, S. Mironov, Phys. Rev. D94, 123516 (2016), 1607.04099

[38] T. Kobayashi, Phys. Rev. D94, 043511 (2016), 1606.05831

[39] S. Akama, T. Kobayashi, Phys. Rev. D95, 064011 (2017), 1701.02926

[40] T. Kobayashi, H. Motohashi, T. Suyama, Phys. Rev. D85, 084025 (2012), [Erratum: Phys. Rev.D96,no.10,109903(2017)], 1202 . 4893

[41] T. Kobayashi, H. Motohashi, T. Suyama, Phys. Rev. D89, 084042 (2014), 1402.6740

[42] O.A. Evseev, O.I. Melichev, Phys. Rev. D97, 124040 (2018), 1711.04152 